ARTICLE

https://doi.org/10.1038/s41467-019-11186-w

\title{
2D semiconductor nonlinear plasmonic modulators
}

\author{
Matthew Klein ${ }^{1,2}$, Bekele H. Badada ${ }^{1}$, Rolf Binder ${ }^{1,2}$, Adam Alfrey ${ }^{1}$, Max McKie ${ }^{1}$, Michael R. Koehler ${ }^{3}$, \\ David G. Mandrus ${ }^{3,4,5}$, Takashi Taniguchi ${ }^{6}$, Kenji Watanabe ${ }^{6}{ }^{6}$, Brian J. LeRoy ${ }^{1}{ }^{1} \&$ John R. Schaibley ${ }^{1}$
}

A plasmonic modulator is a device that controls the amplitude or phase of propagating plasmons. In a pure plasmonic modulator, the presence or absence of a plasmonic pump wave controls the amplitude of a plasmonic probe wave through a channel. This control has to be mediated by an interaction between disparate plasmonic waves, typically requiring the integration of a nonlinear material. In this work, we demonstrate a 2D semiconductor nonlinear plasmonic modulator based on $\mathrm{W} \mathrm{WSe}_{2}$ monolayer integrated on top of a lithographically defined metallic waveguide. We utilize the strong interaction between the surface plasmon polaritons (SPPs) and excitons in the $\mathrm{WSe}_{2}$ to give a $73 \%$ change in transmission through the device. We demonstrate control of the propagating SPPs using both optical and SPP pumps, realizing a 2D semiconductor nonlinear plasmonic modulator, with an ultrafast response time of $290 \mathrm{fs}$.

\footnotetext{
${ }^{1}$ Department of Physics, University of Arizona, Tucson, AZ 85721, USA. ${ }^{2}$ College of Optical Sciences, University of Arizona, Tucson, AZ 85721, USA. ${ }^{3}$ Department of Materials Science and Engineering, University of Tennessee, Knoxville, TN 37996, USA. ${ }^{4}$ Materials Science and Technology Division, Oak Ridge National Laboratory, Oak Ridge, TN 37831, USA. ${ }^{5}$ Department of Physics and Astronomy, University of Tennessee, Knoxville, TN 37996, USA.

${ }^{6}$ National Institute for Materials Science, Tsukuba, Ibaraki 305- 0044, Japan. Correspondence and requests for materials should be addressed to

J.R.S. (email: johnschaibley@email.arizona.edu)
} 
P lasmonic modulators have been highly sought after for approaches to optical frequency information processing devices $^{1-5}$. Optical frequency plasmonic devices offer potential advantages over electronic devices due to the high carrier frequency of optical waves, as well as the potential to use ultrafast solid-state nonlinearities for sub-picosecond switching times. Furthermore, by using plasmonic structures, optical frequency waves can be confined to sub-free-space wavelength waveguides allowing for miniaturization of on-chip optical devices ${ }^{1}$. Early plasmonic modulators demonstrated modulation using quantum $\operatorname{dots}^{3}$, photochromic molecules ${ }^{6}$ as integrated nonlinear materials, but were limited by slow ( $>40 \mathrm{~ns}$ and $10 \mathrm{~s})$ switching times ${ }^{3,6}$. Ultrafast ( 200 fs) plasmonic modulation was demonstrated with a modulation depth of $7.5 \%$ $(\sim 0.3 \mathrm{~dB})$ by direct optical pumping of bare metallic plasmonic waveguides $^{5}$, but required the use of high $\sim 90 \mathrm{~nJ}$ pump pulse energy. The current state-of-the-art plasmonic modulators based on traditional bulk nonlinear materials and nanoplasmonic resonator/interferometer structures typically can achieve modulation depths on order of $1-10 \mathrm{~dB}_{\mu} \mathrm{m}^{-1}$ with response times $>2 \mathrm{ps}$ and require $\sim 3-20 \mathrm{pJ}$ of pulse energy to operate $^{7-9}$. Recently, graphene-based all-optical ${ }^{10}$ and plasmonic $^{11,12}$ modulators have been studied, and compared favorably to previous modulators in terms of switching speed and energy ${ }^{11}$. In 2018, a graphene-based plasmonic modulator achieved $0.2 \mathrm{~dB}_{\mu \mathrm{m}}^{-1}$ modulation depth with a switching energy of $155 \mathrm{fJ}$ and a response time of 2.2 ps using a deep subwavelength plasmonic waveguide ${ }^{12}$. This progress motivates the investigation of other atomically thin materials in plasmonic modulator structures, that have the potential to achieve faster response times, lower switching energies and larger modulation depths.

In free space optical measurements, monolayer $\mathrm{WSe}_{2}$ and other semiconducting TMDs are known to exhibit large light-matter interactions and large third-order nonlinear optical susceptibilities near their exciton resonance ${ }^{13-19}$. Recently, there has been significant interest in using monolayer TMDs for plasmonic applications including the demonstration of SPPs coupling to dark excitons in monolayer $\mathrm{WSe}_{2}{ }^{20,21}$, increasing the nonlinear response using localized plasmonic effects ${ }^{22-24}$, and enhancement of single quantum emitter emission rates 25,26

In this work, we investigate the $2 \mathrm{D}$ semiconductor-plasmonic structure as depicted in Fig. 1a in order to understand the fundamental exciton-SPP interactions and to demonstrate their promise for active plasmonic devices. Our results rely on the atomically thin nature of the TMD and the surface confined SPP mode to realize an attractive geometry where the active layer is near the maximum amplitude of the SPP mode. Furthermore, we develop a novel, self-consistent theory of exciton-SPP (E-SPP) coupling that is unique to the $2 \mathrm{D}$ layer geometry and includes a complete E-SPP dispersion relation for arbitrary distances between the metal surface and the TMD layer. We show that our E-SPP model is highly predictive for both the linear (transmission) and nonlinear (differential transmission) response. We take advantage of the fast nonlinear optical response of $2 \mathrm{D}$ semiconductor excitons to realize an ultra-low switching energy plasmonic modulator with a modulation depth of at least $4.1 \%$ in continuous wave $(\mathrm{CW})$ measurements, limited by the pump power used in the measurement. Our time-domain measurements reveal a fast (slow) component of the nonlinear response with a response time of $290 \mathrm{fs}$ ( $13.7 \mathrm{ps}$ ).

\section{Results}

Fabrication of E-SPP device and linear response. Monolayer $\mathrm{WSe}_{2}$ was integrated on top of the metallic waveguide structures to serve as a nonlinear active layer. Monolayer $\mathrm{WSe}_{2}$ was isolated through mechanical exfoliation from high quality bulk crystals. The $\mathrm{WSe}_{2}$ thickness was confirmed by photoluminescence. In order to electrically isolate the $\mathrm{WSe}_{2}$ from the metallic waveguide (to avoid quenching of excitons), it was encapsulated with hBN. The $\mathrm{hBN}-\mathrm{WSe}_{2}-\mathrm{hBN}$ heterostructures were fabricated and transferred onto the waveguides using a polymer based dry transfer technique (polycarbonate film on polydimethylsiloxane, PDMS, stamp $)^{27}$. The transfer was performed under a microscope based probe station to allow for alignment of the $2 \mathrm{D}$ heterostructure and waveguide. All of the $\mathrm{CW}$ measurements in the main text were performed on the same sample with $5 \mathrm{~nm}$ top and a

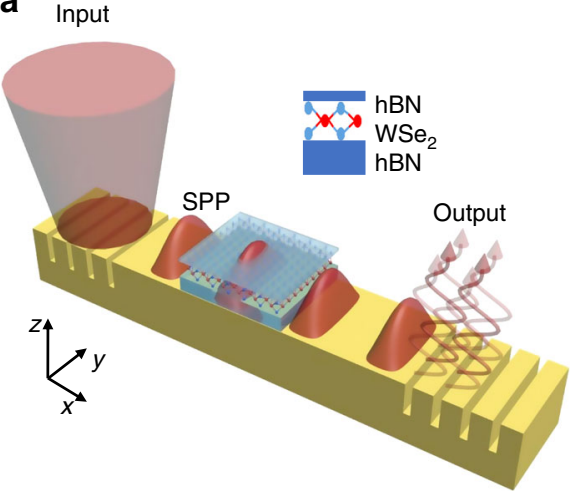

b

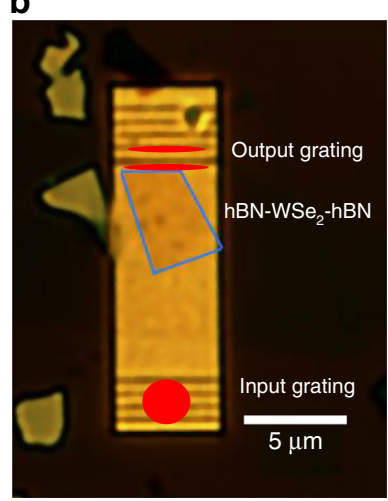

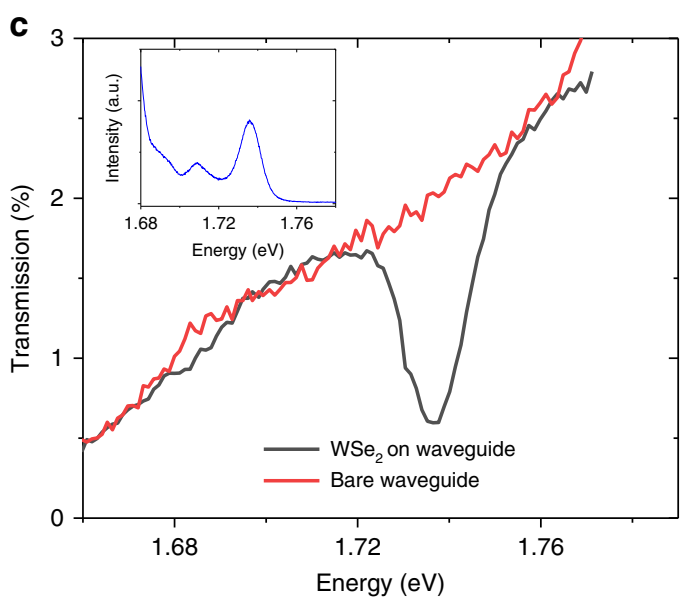

Fig. 1 Plasmonic modulator device and linear response. a Depiction of the 2D material plasmonic device. SPPs are launched at the input of the device by focusing a free space laser onto an input coupler. The SPPs propagate through the waveguide where they can interact with excitons in the active WSe $\mathrm{e}_{2}$ layer, encapsulated in hBN. The SPPs are coupled back to free space photons by an output grating coupler. The upper inset depicts the hBN-WSe $e_{2}-\mathrm{hBN}$ heterostructure on top of the waveguide. The lower inset shows the axes used for the theory and simulations. $\mathbf{b}$ Optical image of the main device used in the experiments. The red dot shows where the grating was illuminated with the input laser, and the red slits show the typical far-field output profile. The blue box shows the location of the active $\mathrm{hBN}-\mathrm{WS} \mathrm{e}_{2}-\mathrm{hBN}$ heterostructure. c Transmission data of the hBN-WSe ${ }_{2}-\mathrm{hBN}$ plasmonic device (black). The normalized transmission of the bare waveguide is shown (red). The inset shows the far-field PL spectrum of the device when excited with a $532 \mathrm{~nm}$ $(2.33 \mathrm{eV})$ laser 

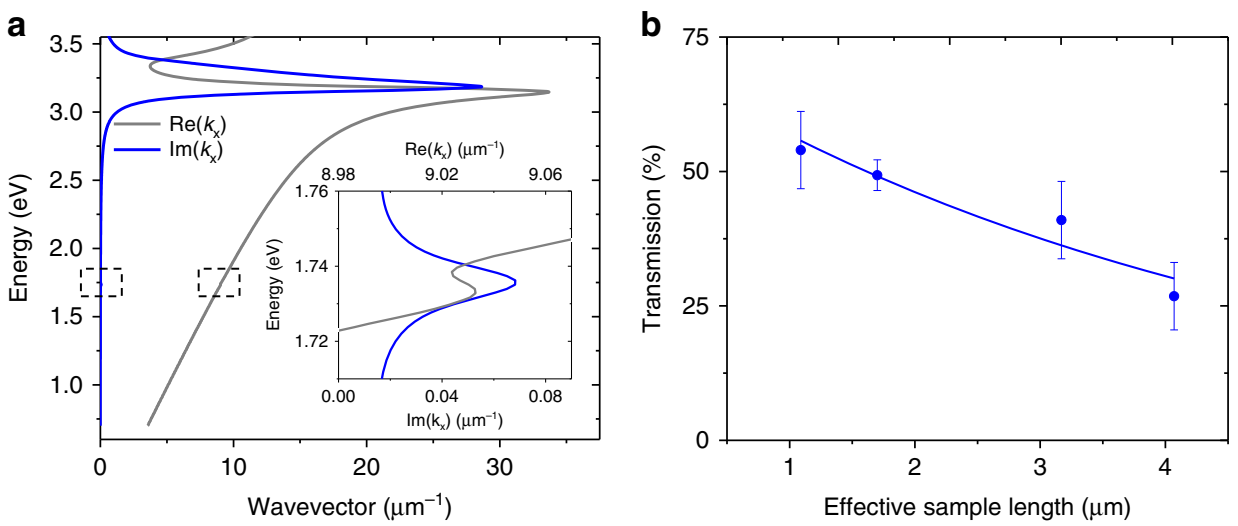

Fig. 2 Linear response of exciton surface plasmon polaritons. a Calculated real (gray) and imaginary (blue) parts of the dispersion relation for exciton surface plasmon polaritons, E-SPPs. The inset shows the dispersion near the exciton resonance ( $1.737 \mathrm{eV})$, whose location is depicted by dashed boxes on the main figure. $\mathbf{b}$ Measured transmission at the exciton resonance as a function of the effective $\mathrm{WSe}_{2}$ sample length on the waveguide

$2 \mathrm{~nm}$ bottom hBN thickness. Time-domain measurements were performed on another sample with $9 \mathrm{~nm}$ and $3 \mathrm{~nm} \mathrm{hBN}$ thickness. An optical microscope image of a hybrid 2D material plasmonic structure is shown in Fig. $1 \mathrm{~b}$.

The hybrid hBN-WSe $\mathrm{h}_{2} \mathrm{hBN} /$ plasmonic structures were measured at $4.5 \mathrm{~K}$ (CW measurements) and $11 \mathrm{~K}$ (time-domain measurements) in a closed-cycle optical cryostat to reduce thermal broadening effects. The transmission spectra and CW nonlinear measurements were measured using two tunable Ti: sapphire continuous wave lasers (M Squared SolsTiS). The laser was focused to a diffraction limited spot on the input grating coupler. Light scattered from the output grating coupler was isolated using a spatial filter and measured with a silicon photodiode. In the linear transmission measurements, the probe laser was modulated for lock-in detection. In the nonlinear spectroscopy measurements, pump and probe beams were amplitude modulated at different frequencies near $500 \mathrm{kHz}$ to allow for lock-in detection at the modulation difference frequency. The time-domain pump-probe measurements were performed with a tunable mode-locked Ti:sapphire laser with a repetition rate of $76 \mathrm{MHz}$ and pulse width of $\sim 120 \mathrm{fs}$.

The SPP transmission spectrum is shown in Fig. 1c for $60 \mu \mathrm{W}$ input power. The black data show the transmission spectrum for the hybrid $\mathrm{hBN}-\mathrm{WSe}_{2}-\mathrm{hBN} /$ plasmonic structure (with a $\sim 4-\mu \mathrm{m}$ long $\mathrm{WSe}_{2}$ layer), and the red data show a reference bare waveguide. At the exciton resonance $(1.737 \mathrm{eV}, 713.6 \mathrm{~nm})$, the transmission is reduced by $\sim 73 \%$ due to the presence of the $\mathrm{WSe}_{2}$ layer, indicating a large interaction between SPPs and $\mathrm{WSe}_{2}$ excitons. By comparing these transmission data to the photoluminescence spectrum (Fig. 1c inset), we can identify the dip in the SPP transmission as originating from the $\mathrm{WSe}_{2}$ neutral exciton $\left(\mathrm{X}^{0}\right)^{14}$. We note that the center energy of the PL and SPP absorption response are aligned to within $1 \mathrm{meV}$, consistent with previous optical measurements on monolayer $\mathrm{WSe}_{2}{ }^{14}$.

Theory of E-SPP. In order to understand the coupling between SPPs and excitons, we use an extension of the well-known SPP dispersion $\left(k_{x}(\omega)\right)$ that relates the wave vector component $k_{x}$, where the axis $x$ is shown in Fig. 1a, of a mode propagating along the surface to its energy ( $\hbar \omega)$ where $\omega$ is the SPP's angular frequency. Our approach complements other theories such as coupled oscillator (plexciton) ${ }^{28,29}$, scattering ${ }^{30}$, and gain-assisted SPP theories ${ }^{4,31,32}$. It is formulated for arbitrary distances between the metal surface and the TMD layer, and reduces to an expression calculated previously 33,34 in the limit of vanishing distance. Using the dielectric function of the metal $\varepsilon_{m}(\omega)$ and the optical susceptibility $\chi(\omega)$ of the TMD layer, we obtain their coupling directly by solving the dispersion relation, which is free of any fitting parameters. We use subscripts 1,2 , and 3 to denote the region above the TMD layer, between the metal surface and the TMD layer, and inside the metal, respectively (Supplementary Fig. 1).

The dispersion relation of the coupled exciton surface plasmon polariton is obtained in a way that is analogous to deriving that of an SPP, i.e., looking for non-trivial solutions of Maxwell's equations that satisfy the continuity relations at the surface and decay away from it. The difference is the presence of the TMD layer, which requires additional continuity relations to be fulfilled, and there is no exponential decay in the region between the metal surface and the TMD layer. The coupling strength between the surface plasmon and the exciton in the TMD layer is governed by the factor $e^{-\operatorname{Im} k_{2 z} z_{\ell}}$ where $k_{2 z}$ is the wave vector component normal to the surface in region 2 , and $z_{\ell}$. As expected, the coupling is strong only if the layer is within the region of the evanescent surface mode. In the limit $z_{\ell} \rightarrow 0$, we obtain $k_{2 z}\left(\varepsilon_{m} k_{2 z}-k_{3 z}\right)+g k_{3 z}=0$ where $k_{i z}= \pm \sqrt{\omega^{2} \varepsilon_{i} / c^{2}-k_{x}^{2}}$ is the wave vector component perpendicular to the surface, the dielectric functions are $\varepsilon_{1}=\varepsilon_{2}=1$ for the vacuum regions and $\varepsilon_{3}=\varepsilon_{m}$, and $g=4 \pi i\left(\omega^{2} / c^{2}-k_{x}^{2}\right) \chi(\omega)$ provides the coupling, in agreement with previous works ${ }^{33,34}$. The more general form where the distance is an arbitrary input parameter is given (Supplementary Note 1). We use a Drude model for the metal and a Lorentz model for the TMD exciton. This results in an ESPP resonance at the exciton energy $(1.737 \mathrm{eV})$ with the real and imaginary parts of $k_{x}(\omega)$ shown in (gray and blue curves, Fig. 2a). The E-SPP group velocity is plotted (Supplementary Fig. 2). The peak value for $\operatorname{Imk}_{\mathrm{x}}$ of $0.07 \mu \mathrm{m}^{-1}$ corresponds to an absorption length of $7.1 \mu \mathrm{m}$. Figure $2 \mathrm{~b}$ shows the measured transmission as a function of the effective $\mathrm{WSe}_{2}$ sample length for the three different structures we investigated (Supplementary Fig. 3a-f). The effective $\mathrm{WSe}_{2}$ sample lengths were estimated from the optical microscope images by calculating an average length over the central $3 \mu \mathrm{m}$ of the waveguide corresponding the full-width half-max of the SPP spatial mode. An exponential fit to these data yields an effective decay length of $4.8 \pm 0.6 \mu \mathrm{m}$, which is in good agreement (within a factor of two) of our theoretical model.

Nonlinear E-SPP interactions. The transmission of the plasmonic device can be controlled by optically pumping the $\mathrm{WSe}_{2}$ excitons, partially saturating the absorption. Figure 3 a depicts the experimental configuration where SPPs propagating through the plasmonic structure serve as the probe, and a free space laser 
a

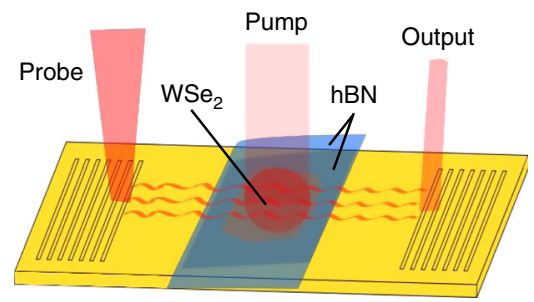

b
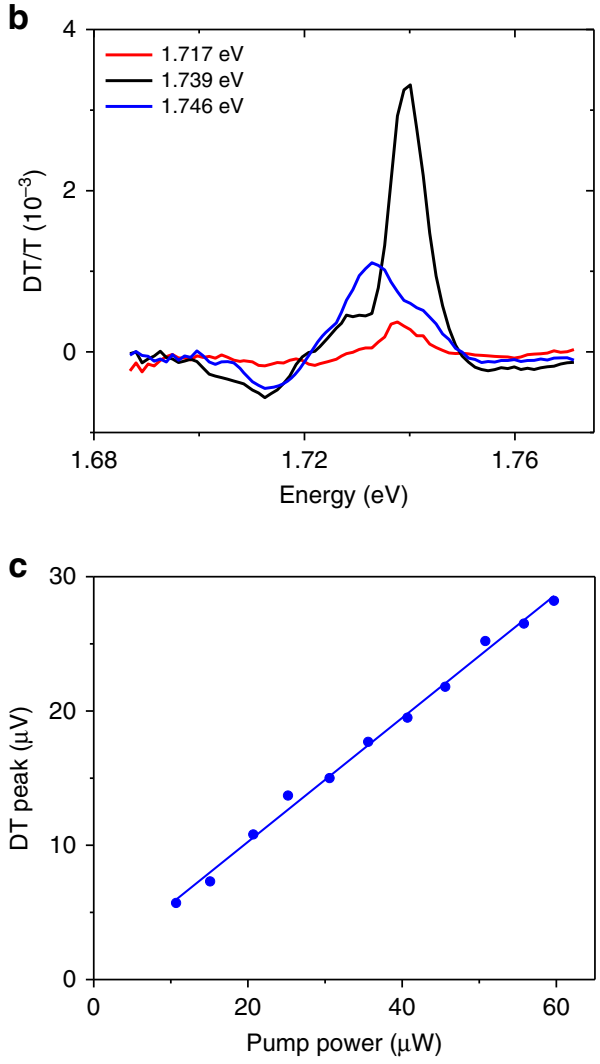

Fig. 3 Optical control of SPP propagation. a Depiction of the photon-SPP pump-probe measurements. SPPs propagating through the device interact with excitons in the $\mathrm{WSe}_{2}$ layer. A free space laser illuminating the $\mathrm{WSe}_{2}$ controls the SPP propagation by saturating the exciton absorption. $\mathbf{b} D T / T$ measurements for three pump photon energies. c Pump power dependence of the DT signal near the peak of the exciton response (1.739 eV pump, $1.743 \mathrm{~nm}$ probe)

focused on the $\mathrm{hBN}-\mathrm{WS} \mathrm{e}_{2}-\mathrm{hBN}$ structure serves as an optical pump. Here, the focused optical pump beam diameter was chosen so that it illuminated nearly the entire $\mathrm{WSe}_{2}$ region with an intensity of $8.5 \times 10^{6} \mathrm{Wm}^{-2}$. Figure $3 \mathrm{~b}$ shows the DT/T spectrum as a function of probe wavelength, i.e., the pump-induced differential transmission (DT) normalized by the probe transmission (T). DT/T spectra for three different pump energies of $1.717 \mathrm{eV}$ (red), $1.739 \mathrm{eV}$ (black), and 1.746 (blue) are shown. When the pump laser is near resonance with the $\mathrm{WSe}_{2} \mathrm{X}^{0}$, the DT/T signal is maximized giving a peak value of $\mathrm{DT} / \mathrm{T}=4.1 \times 10^{-3}$. Figure $3 \mathrm{c}$ shows the pump power dependence of the DT signal near the center of the exciton peak (1.739 eV pump, $1.743 \mathrm{~nm}$ probe). The DT signal is linear with pump power indicating that the DT response arises from the third-order nonlinear susceptibility.

In order to demonstrate plasmonic modulation, we performed nonlinear measurements where both pump and probe lasers were coupled into the input grating, launching pump and probe SPPs (depicted in Fig. 4a). Figure 4b shows the CW DT/T spectra for three different pump-SPP energies $1.715 \mathrm{eV}$ (red), $1.739 \mathrm{eV}$ (black), and $1.771 \mathrm{eV}$ (blue). We again observe a strong nonlinear response at the $\mathrm{X}^{0}$ resonance, corresponding to a maximum DT/ $\mathrm{T}=4.1 \times 10^{-2}$. For this case, we see the DT/T amplitude increases by a factor of 10 over the optically pumped signal. The SPP pump power (of the SPP) dependence is also linear (Fig. 4c), consistent with a third-order nonlinear response. From the finite difference time-domain (FDTD) model, we find that the SPP pump intensity is $4.6 \times 10^{6} \mathrm{Wm}^{-2}, \sim 2$ times smaller than the optical pump case.

In order to quantify the response time of the system, we carried out resonant time-domain pump-probe measurements. We used a single $(\sim 120 \mathrm{fs})$ pulsed laser tuned to resonance $(1.736 \mathrm{eV}, 714$ $\mathrm{nm}$ ) split into pump and probe, and a mechanical delay line to vary the pump-probe temporal separation. The time dependent DT/T response is shown in Fig. 4d. A biexponential is fit to the positive time signal resulting in fast $290 \pm 20 \mathrm{fs}$ and slower $13.7 \pm$ $0.6 \mathrm{ps}$ components to the decay time. We note that these decay times are 5-10 times faster than previously reported for monolayer $\mathrm{WSe}_{2}$ on $\mathrm{SiO}_{2}{ }^{35,36}$ which is not surprising due to the coupling to the SPP mode. Figure 4e shows the DT response as a function of pump pulse energy (of the SPP). We note that the required pump pulse energy to achieve a DT/T $\approx 1 \%$ is $650 \mathrm{fJ}$. We also note that in SPP pump-SPP probe measurements both pump and probe lasers were detected simultaneously, which both contribute to the DT signal. To account for this, the transmission used to calculate DT/T is the sum of both pump and probe beams combined.

To understand this plasmonic modulation effect and to estimate the order-of-magnitude of the third-order nonlinearity, we extended our linear analysis (Fig. 2a) to the third-order nonlinear response with perturbation theory. Since we only observe significant signal near $1.737 \mathrm{eV}$, we limit our model to inplane dipoles associated with the $\mathrm{X}^{0}$ excitons. This reduces the susceptibility tensor to the single third-order component $\chi^{(3)}$ (Supplementary Note 1). We assume that the pump-induced change in the susceptibility $(\Delta \chi)$ is proportional to the average pump intensity $\left(I_{p}\right)$, i.e. $\Delta \chi\left(\omega, I_{\mathrm{p}}\right) \approx \frac{4 \pi}{c} I_{\mathrm{p}} \chi^{(3)}(\omega)$. We can then use the linear dispersion relation with the replacement $\chi(\omega) \rightarrow \chi$ $\left(\omega, I_{\mathrm{p}}\right)=\chi(\omega)+\Delta \chi\left(\omega, I_{\mathrm{p}}\right)$ which yields a pump-induced change in the dispersion, $\Delta k_{x}$, and thus a measure of the pump-induced differential transmission DT/T. We deduce a value for $\operatorname{Im} \chi^{(3)}$ at the peak of the E-SPP resonance by using the experimental value for DT/T and the estimated average intensity. For both, optical pump/SPP probe and SPP pump/SPP probe we find the order of magnitude of $\operatorname{Im} \chi^{(3)}$ to be $-10^{-20} \mathrm{~m}^{3} \mathrm{~V}^{-2}$, in agreement with previously reported all-optical experiments ${ }^{16,35}$. We note this value is a $2 \mathrm{D}$ third-order susceptibility. To compare this value to a hypothetical 3D susceptibility, one must divide it by the monolayer thickness.

\section{Discussion}

In this work, we have investigated both the linear and nonlinear response of excitons interacting with propagating SPPs in metallic waveguide structures. We show that the linear absorption of SPPs can be very large, exceeding $73 \%$. The large absorption and nonlinear response might be surprising considering that the outof-plane spatial extent of an SPP $(\sim 400 \mathrm{~nm})$ is much larger than the TMD thickness $(0.7 \mathrm{~nm})$. However, our theoretical analysis is consistent with the measurements yielding an absorption coefficient on the order of $0.2 \mu \mathrm{m}^{-1}$. The key to the large linear absorption is the nanometer-scale proximity of the TMD layer to the metal surface, which allows for the active layer to be located near the maximum of the SPP mode. By performing both optical pump and SPP pump DT measurements, we demonstrate control 
a

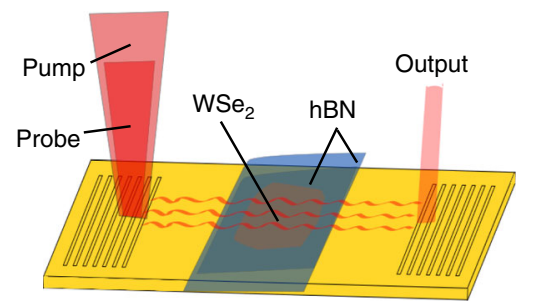

b

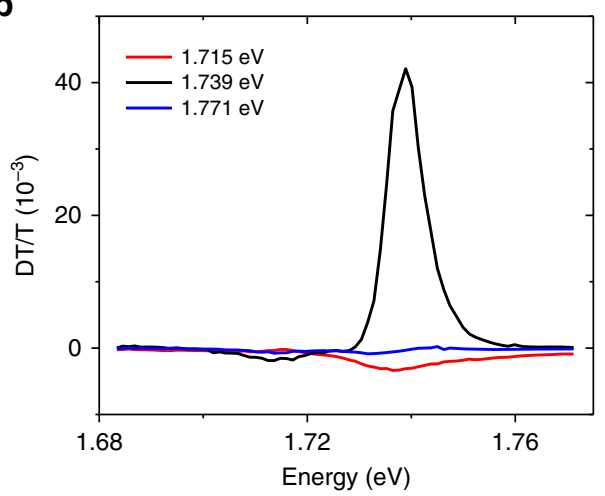

C

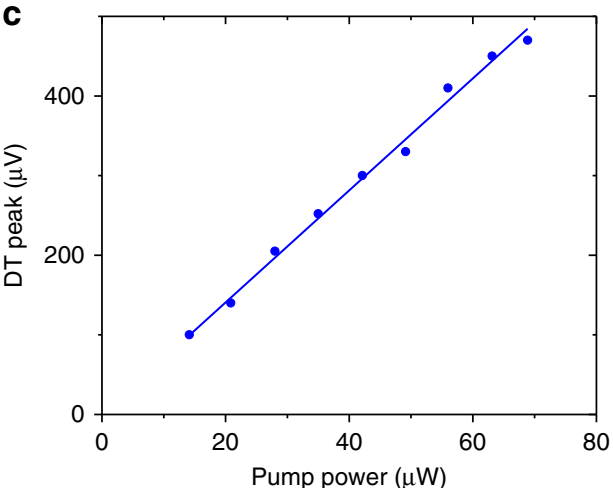

d

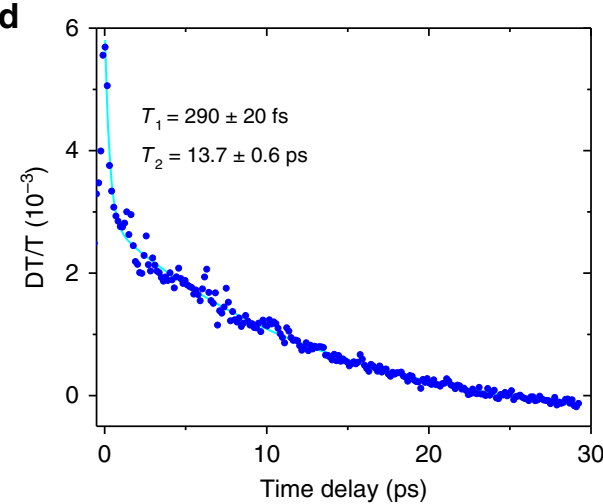

e

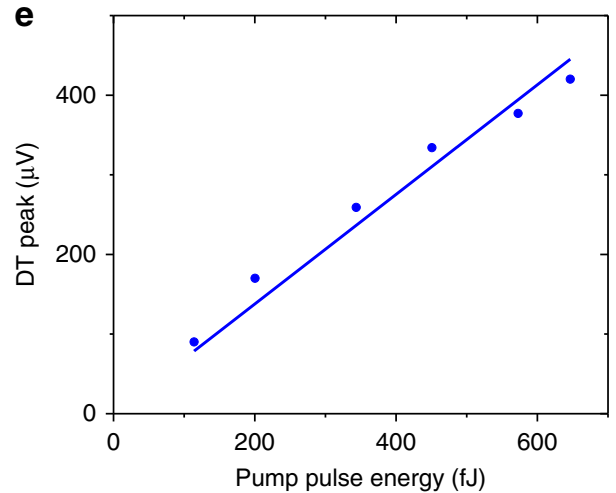

Fig. 4 SPP control of SPP propagation. a Depiction of the SPP-SPP pump-probe measurements. Pump and probe SPPs are launched at the input grating coupler. SPPs propagating through the device interact with excitons in the $\mathrm{WSe}_{2}$ layer. In this plasmonic modulator configuration, the pump-SPP saturates the exciton absorption, resulting in an increase in probe SPP transmission. b SPP pump energy dependence of the DT/T spectrum. c SPP pump power dependence of the DT signal near the peak of the exciton response (pump $1.739 \mathrm{eV}$, probe $1.741 \mathrm{eV}$ ). $\mathbf{d}$ Time resolved DT/T response for a similar device measured at $11 \mathrm{~K}$ with a photon energy of $1.736 \mathrm{eV}$. The blue points are the data and the cyan curve is a biexponential fit whose time constants are shown. e SPP pump pulse energy dependence of the pulsed DT response measured near zero time delay

of SPP propagation with a DT/T response exceeding $4 \%$. The modulation depth per unit length achieved in our modulator $\left(0.04 \mathrm{~dB} \mu \mathrm{m}^{-1}\right)$ is within an order of magnitude of other state-ofthe-art plasmonic modulators based on monolayer graphene ${ }^{12}$. We note that in both optical and SPP pumped measurements, the maximum pump powers we used were conservatively chosen to avoid sample damage. Since the DT signals are linear in pump intensity up to the highest pump powers used (Figs. 3c and 4c), the reported modulation depths should be taken as lower bounds on the achievable modulation depth.

In principle, the modulation depth could be further enhanced by using longer TMD layers, stacking several TMD layers separated by $\mathrm{hBN}$, or by decreasing the SPP mode size by depositing a high dielectric constant material on top of the structure. The modulation depth can also be increased by utilizing an interferometric or slot waveguide modulator structure ${ }^{11,12}$. Furthermore, our theory predicts that the modulation depth increases with decreasing detuning between the exciton and the SPP resonance. This plasmonic enhancement follows from the equation $\Delta k_{x}=h_{\mathrm{enh}}(\omega) \Delta \chi(\omega)$, where the nonlinear change in the complex-valued propagation vector $\Delta k_{x}$, which governs directly measurable quantities such as DT/T $\left(\propto \operatorname{Im} \Delta \mathrm{k}_{\mathrm{x}}\right)$ is related to the change in the susceptibility $\Delta \chi(\omega)$. The plasmonic enhancement factor $h_{\text {enh }}(\omega)$ is shown (Supplementary Fig. 4). Our model shows that increasing the exciton energy to $2.8 \mathrm{eV}$ (with all other parameters unchanged) would increase $\Delta k_{x}$ by more than 2 orders of magnitude.

To further quantify the performance of our modulator relative to previous works, we consider the response time and minimum energy needed to switch the modulator. Indeed the fast component of our response ( $290 \mathrm{fs}$ ) is comparable to the fastest previously reported plasmonic modulators ${ }^{5}$, but we achieve a similar modulation depth with a $\sim 10^{5}$ times lower pump pulse energy. Compared to other state-of-the-art plasmonic modulators which typically require $\sim 3-20 \mathrm{pJ}$ of pulse energy to operate, with response times $>2 \mathrm{ps}^{7-9}$, our demonstration of a 290 -fs response time with $650 \mathrm{fJ}$ pump pulse energy compares favorably. We note that assuming a Gaussian pulse, this response time corresponds to 
a modulation bandwidth $\sim 1.5 \mathrm{THz}$. We believe that future $2 \mathrm{D}$ semiconductor-plasmonic structures based on our reported nonlinear exciton-SPP plasmonic modulation effect could pave the way towards ultrafast plasmonic amplifiers and transistors with ultra-low switching energies.

\section{Methods}

Fabrication. The gold waveguide was fabricated on $285 \mathrm{~nm} \mathrm{SiO} / 2 \mathrm{Si}$ substrates by a two-step electron beam lithography process using an electron beam lithography system (100 kV Ellionix) and a spin coated poly(methyl methacrylate), PMMA, resist. In the first step, $200 \mathrm{~nm}$ gold was (electron beam) evaporated onto the substrate using $10 \mathrm{~nm}$ titanium sticking layer. In the second lithography step, PMMA was respun and the grating pattern was written and developed. We used an $\mathrm{Ar}^{+}$milling process to etch the grating couplers into the waveguide. The waveguides are $5 \mu \mathrm{m} \times 13 \mu \mathrm{m}$. The grating couplers are composed of five grooves that are $40 \mathrm{~nm}$ deep with a width of $110 \mathrm{~nm}$ and period of $570 \mathrm{~nm}$. The bare waveguides were characterized using atomic force microscopy (Supplementary Fig. 5a-d) and optical spectroscopy (Fig. 1c). The waveguide and grating coupler designs were optimized using an FDTD model. Simulations of the bare metallic structure show a maximum transmission of $\sim 4 \%$ at the exciton resonance (Supplementary Fig. 6). We integrate a hexagonal boron nitride (hBN) encapsulated monolayer transition metal dichalcogenide (TMD) semiconductor, $\mathrm{WSe}_{2}$, on top of the waveguide (see Fig. 1a), where the interaction between SPPs and excitons in the $\mathrm{WSe}_{2}$ provides the nonlinear response needed for modulation.

\section{Data availability}

The data that support the findings of this study are available from the corresponding author upon reasonable request.

Received: 19 December 2018 Accepted: 20 June 2019

Published online: 22 July 2019

\section{References}

1. Atwater, H. A. The promise of plasmonics. Sci. Am. 296, 56-62 (2007),

2. Krasavin, A. V., Vo, T. P., Dickson, W., Bolger, P. M. \& Zayats, A. V. Allplasmonic modulation via stimulated emission of copropagating surface plasmon polaritons on a substrate with gain. Nano Lett. 11, 2231-2235 (2011).

3. Pacifici, D., Lezec, H. J. \& Atwater, H. A. All-optical modulation by plasmonic excitation of CdSe quantum dots. Nat. Photon. 1, 402-406 (2007).

4. Li, D. \& Ning, C. Giant modal gain, amplified surface plasmon-polariton propagation, and slowing down of energy velocity in a metal-semiconductormetal structure. Phys. Rev. B 80, 153304 (2009).

5. MacDonald, K. F., Sámson, Z. L., Stockman, M. I. \& Zheludev, N. I. Ultrafast active plasmonics. Nat. Photon. 3, 55-58 (2009).

6. Pala, R. A., Shimizu, K. T., Melosh, N. A. \& Brongersma, M. L. A nonvolatile plasmonic switch employing photochromic molecules. Nano Lett. 8, 1506-1510 (2008).

7. Schönenberger, S. et al. Ultrafast all-optical modulator with femtojoule absorbed switching energy in silicon-on-insulator. Opt. Express 18, 22485-22496 (2010)

8. Nielsen, M. \& Elezzabi, A. Ultrafast all-optical modulation in a silicon nanoplasmonic resonator. Opt. Express 21, 20274-20279 (2013).

9. Neira, A. D., Wurtz, G. A., Ginzburg, P. \& Zayats, A. V. Ultrafast all-optical modulation with hyperbolic metamaterial integrated in Si photonic circuitry. Opt. Express 22, 10987-10994 (2014)

10. Li, W. et al. Ultrafast all-optical graphene modulator. Nano Lett. 14, 955-959 (2014).

11. Zhou, F. \& Du, W. Ultrafast all-optical plasmonic graphene modulator. Appl. Opt. 57, 6645-6650 (2018).

12. Ono, M. et al. Ultrafast and energy-efficient all-optical modulator based on deep-subwavelength graphene-loaded plasmonic waveguides, in Conference on Lasers and Electro-Optics, OSA Technical Digest (2018)

13. Mak, K. F., Lee, C., Hone, J., Shan, J. \& Heinz, T. F. Atomically thin $\mathrm{MoS}_{2}$ : a new direct-gap semiconductor. Phys. Rev. Lett. 105, 136805 (2010).

14. Jones, A. M. et al. Optical generation of excitonic valley coherence in monolayer WSe $\mathrm{W}_{2}$. Nat. Nanotechnol. 8, 634-638 (2013).

15. Splendiani, A. et al. Emerging photoluminescence in monolayer $\mathrm{MoS}_{2}$. Nano Lett. 10, 1271-1275 (2010)

16. Schaibley, J. R. et al. Population pulsation resonances of excitons in monolayer $\mathrm{MoSe}_{2}$ with sub-1 $\mu \mathrm{eV}$ linewidths. Phys. Rev. Lett. 114, 137402 (2015).

17. Back, P., Zeytinoglu, S., Ijaz, A., Kroner, M. \& Imamoğlu, A. Realization of an electrically tunable narrow-bandwidth atomically thin mirror using monolayer $\mathrm{MoSe}_{2}$. Phys. Rev. Lett. 120, 037401 (2018).
18. Ross, J. S. et al. Electrical control of neutral and charged excitons in a monolayer semiconductor. Nat. Commun. 4, 1474 (2013).

19. Scuri, G. et al. Large excitonic reflectivity of monolayer $\mathrm{MoSe}_{2}$ encapsulated in hexagonal boron nitride. Phys. Rev. Lett. 120, 037402 (2018).

20. Zhou, Y. et al. Probing dark excitons in atomically thin semiconductors via near-field coupling to surface plasmon polaritons. Nat. Nanotechnol. 12, 856 (2017).

21. Park, K.-D., Jiang, T., Clark, G., Xu, X. \& Raschke, M. B. Radiative control of dark excitons at room temperature by nano-optical antenna-tip Purcell effect. Nat. Nanotechnol. 13, 59 (2018).

22. Kleemann, M.-E. et al. Strong-coupling of $\mathrm{WSe}_{2}$ in ultra-compact plasmonic nanocavities at room temperature. Nat. Commun. 8, 1296 (2017).

23. Abid, I. et al. Resonant surface plasmon-exciton interaction in hybrid $\mathrm{MoSe}_{2}$ Au nanostructures. Nanoscale 8, 8151-8159 (2016).

24. Johnson, A. D., Cheng, F., Tsai, Y. \& Shih, C.-K. Giant enhancement of defectbound exciton luminescence and suppression of band-edge luminescence in monolayer $\mathrm{WSe}_{2}-\mathrm{Ag}$ plasmonic hybrid structures. Nano Lett. 17, 4317-4322 (2017).

25. Tran, T. T. et al. Deterministic coupling of quantum emitters in $2 \mathrm{D}$ materials to plasmonic nanocavity arrays. Nano Lett. 17, 2634-2639 (2017).

26. Cai, T. et al. Coupling emission from single localized defects in twodimensional semiconductor to surface plasmon polaritons. Nano Lett. 17, 6564-6568 (2017).

27. Zomer, P., Guimarães, M., Brant, J., Tombros, N. \& van Wees, B. Fast pick up technique for high quality heterostructures of bilayer graphene and hexagonal boron nitride. Appl. Phys. Lett. 105, 013101 (2014).

28. Schlather, A. E., Large, N., Urban, A. S., Nordlander, P. \& Halas, N. J. Nearfield mediated plexcitonic coupling and giant Rabi splitting in individual metallic dimers. Nano Lett. 13, 3281-3286 (2013).

29. Held, M. et al. Ultrastrong coupling of electrically pumped near-infrared exciton-polaritons in high mobility polymers. Adv. Opt. Mater. 6, 1700962 (2018).

30. Fofang, N. T. et al. Plexcitonic nanoparticles: plasmon- exciton coupling in nanoshell- J-aggregate complexes. Nano Lett. 8, 3481-3487 (2008).

31. Maier, S. A. \& Atwater, H. A. Plasmonics: localization and guiding of electromagnetic energy in metal/dielectric structures. J. Appl. Phys. 98, 10 (2005).

32. Nezhad, M. P., Tetz, K. \& Fainman, Y. Gain assisted propagation of surface plasmon polaritons on planar metallic waveguides. Opt. Express 12, 4072-4079 (2004)

33. Jablan, M., Buljan, H. \& Soljačić, M. Plasmonics in graphene at infrared frequencies. Phys. Rev. B 80, 245435 (2009).

34. Gonçalves, P., Bertelsen, L., Xiao, S. \& Mortensen, N. A. Plasmon-exciton polaritons in two-dimensional semiconductor/metal interfaces. Phys. Rev. B 97, 041402 (2018)

35. Aivazian, G. et al. Many-body effects in nonlinear optical responses of 2D layered semiconductors. 2D Materials 4, 025024 (2017).

36. Moody, G. et al. Intrinsic homogeneous linewidth and broadening mechanisms of excitons in monolayer transition metal dichalcogenides. Nat. Commun. 6, 8315 (2015).

\section{Acknowledgements}

We acknowledge useful discussions with Javier García de Abajo, Robert Norwood, Josh Hendrickson, and Ricky Gibson. We thank Ty Newhouse-Illige and Weigang Wang for performing the $\mathrm{Ar}^{+}$milling process. We thank Fateme Mahdikhanysarvejahany for assistance in performing the time-domain measurements. This work is mainly supported by the AFOSR-YIP award No. FA9550-17-1-0215. M.R.K. and D.G.M. acknowledge support from the Gordon and Betty Moore Foundation's EPiQS Initiative through grant GBMF4416. K.W. and T.T. acknowledge support from the Elemental Strategy Initiative conducted by the MEXT, Japan and the CREST (JPMJCR15F3), JST. J.R.S. acknowledges support from The Science Foundation of Arizona, Bisgrove Scholars Program (Grant No. BSP 0821-17) and AFOSR (Grant No. FA9550-18-1-0049). B.J.L. acknowledges support from the National Science Foundation under Grant No. EECS-1607911. AFM images and data were collected in the W.M. Keck Center for Nano-Scale Imaging in the Department of Chemistry and Biochemistry at the University of Arizona using equipment supported by the National Science Foundation under Grant No. 1337371. We would also like to thank Park Systems for their long term loan of the NX20 AFM instrument.

\section{Author contributions}

J.R.S. conceived and supervised the project. M.K. and B.H.B. fabricated the devices and performed the experiments, assisted by A.A. and M.M.; M.K., B.H.B., and J.R.S. analyzed the data with input from B.J.L.; M.R.K. and D.G.M. provided and characterized the bulk $\mathrm{WSe}_{2}$ crystals. T.T. and K.W. provided hBN crystals. R.B. developed and evaluated the coupled exciton-SPP (E-SPP) theory. M.K., B.H.B., R.B., and J.R.S. wrote the paper. All authors discussed the results. 


\section{Additional information}

Supplementary Information accompanies this paper at https://doi.org/10.1038/s41467019-11186-w.

Competing interests: The authors declare no competing interests.

Reprints and permission information is available online at http://npg.nature.com/ reprintsandpermissions/

Peer review information: Nature Communications thanks Jason Smith and other anonymous reviewers for their contribution to the peer review of this work.

Publisher's note: Springer Nature remains neutral with regard to jurisdictional claims in published maps and institutional affiliations. (c) (i) Open Access This article is licensed under a Creative Commons Attribution 4.0 International License, which permits use, sharing, adaptation, distribution and reproduction in any medium or format, as long as you give appropriate credit to the original author(s) and the source, provide a link to the Creative Commons license, and indicate if changes were made. The images or other third party material in this article are included in the article's Creative Commons license, unless indicated otherwise in a credit line to the material. If material is not included in the article's Creative Commons license and your intended use is not permitted by statutory regulation or exceeds the permitted use, you will need to obtain permission directly from the copyright holder. To view a copy of this license, visit http://creativecommons.org/ licenses/by/4.0/.

(C) The Author(s) 2019 